\title{
New Algorithms for Radiative Transfer in Accretion Disks and Surroundings
}

\author{
Guido Kanschat
}

The simulation of astrophysical radiative transfer problems (RTP) is a hard task. On the one hand, the high dimension of the computational domain ( 3 space, 2 ordinate and 1 frequency variables) calls for huge amounts of memory even for coarse discretisations. On the other hand, accretion discs show very localised features, making a high resolution in some regions of the disk necessary. Furthermore, there should be an estimate of the numerical errors to avoid physical misinterpretation.

Apart from the usage of parallel machines with their big memory to enable the computation of RTP's, we developed a means to generate nearly optimal discretisation meshes. Combined with efficient error estimates, we can solve RTP's to a very high guaranteed accuracy. These estimates, weighted residual a posteriori error estimates, can not only control global error quantities like the mean quadratic error or the error in the energy norm. They can be suited to nearly any error functional. A typical observer situation in astrophysics is an object seen as a point source located at infinity. The simulated value to be compared with observations therefore is

$$
\Psi(I)=-\int_{\Gamma_{+}} I\left(x,-\omega_{\mathrm{Obs}}\right) n_{\omega_{\mathrm{Ob}} \mathrm{s}} \cdot n_{\Gamma} d \sigma
$$

the integral of the intensity $I$ over the visible part $\Gamma_{+}$of the boundary of the object, weighted by the scalar product of the viewing direction $\omega_{\mathrm{Obs}}$ and the outer surface normal $n_{\Gamma}$. For this quantity, the solution of a so called dual problem is computed. This is used to measure the influence of the error at a point on the error functional $\Psi$. While control of the mean quadratic error is impossible due to computation overhead, these estimates provide sharp error bounds overestimating the true error by a factor lower than four. Therefore, they are suitable to guarantee reliability of the numerical solution to the radiative transfer equation in complex geometries.

The crucial point in the development of those error estimates is Galerkin orthogonality, a special error representation of so called Galerkin methods like finite elements (FE). To exploit this powerful means, we apply the finite element method (FEM) to the radiative transfer equation. Considering the scattering term, the standard FEM with piecewise constant trial functions is well suited. Since it is equivalent to the discrete ordinate method, it avoids the overhead calculating matrix elements, which arises usual in the FE discretisation of integral operators.

Standard FEM is not applicable to advection problems, since it lacks the necessary stability. Therefore, the advection operator $n_{\omega} \cdot \nabla I$ is discretised using the finite element streamline diffusion method (SDFEM). SDFEM has superior 
approximation properties compared to standard schemes. Opposed to upwind schemes, it conserves the Galerkin method property of FEM.

An additional advantage of FEM is the inherent possibility of the easy use of solution adaptive grids. These are constructed by using the error estimators mentioned above in a local mesh refinement algorithm. The grids obtained by this process allow the solution of radiative transfer problems up to a given error tolerance with optimal usage of computing resources. We would like to stress, that the grids are not only optimised to the data and solution of the problem, but also to the kind of value we want to get out of the simulation.

The solution of the discrete systems is accomplished by a combination of an adaptive multi-grid scheme of optimal complexity and non-symmetric Krylovspace methods like GMRES or Bicgstab. These methods exploit to a very high extend the special eigenvalue structure of RTP's.

The high dimensionality of radiative transfer problems makes the usage of parallel computers inevitable for reasons of computing time as well as memory requirements. We distribute the ordinate space on multiple processors. This allows us to invert the advection operator on a purely local basis. Data exchange is necessary for the computation of scattering only. The efficiency of this method is bounded from zero by a machine dependent number ranging between 0.2 on machines with slow communication hardware up to more than 0.9 on faster ones.

A more elaborate description of the methods mentioned, as well as an additional bibliography can be found in Kanschat (1996).

\section{References}

Kanschat, G. Dissertation, Universität Heidelberg, 1996. 